\title{
Control of xylogenesis within the whole tree
}

\author{
R. Aloni \\ Department of Botany, The George S. Wise Faculty of Life Sciences, Tel Aviv University, Tel Aviv \\ 69978, Israel
}

\section{Introduction}

The problems to be dealt with here concern the control of organized differentiation of the wood within a whole tree. Anatomically, the wood is the xylem and it has a different structure and composition in different parts of the tree. In mature organs, the wood is produced by a lateral meristem, the vascular cambium. The wood produced by the vascular cambium is termed secondary xylem. It is a complex tissue, comprised of several types of cells. Xylem differentiation within the plant is characterized by a high degree of order. The vascular and supporting elements, as well as the parenchymatic cells, differentiate in a predictable, three-dimensional structure, typical for each species. Therefore, a tree species can be identified solely by its xylem. Xylogenesis within the whole tree is an open type of differentiation, which continues as long as the tree grows by apical and lateral meristems. Thus, there is a continuous development of new xylem tissues that are in a dynamic relationship with one another. This complex developing system raises many questions about control mechanisms, and these have enormous economic impor- tance in terms of improving wood quality and forest productivity.

Wood quality depends upon the form and size of a tree, as well as on the wood's anatomical structure and chemical composition. The quality of an isolated piece of wood is the result of the quantitative relationship between the type of xylem cells, their size and density, as well as their physical structure and chemical composition. The problems to be dealt with here will be restricted to the control of xylem development and structure from the level of individual cells to that of the entire plant. In order to clarify how the wood, within the whole tree, is being formed in an orderly manner, I present current thoughts and evidence on the role of both endogenous and exogenous factors, including gall-forming aphids, in controlling the organized differentiation of xylem in plants, especially in forest trees.

\section{Control of xylogenesis by auxin flow}

Developing buds and young growing leaves induce vascular differentiation which extends from the developing leaves toward the root tips. The auxin, indole-3- 
acetic acid (IAA), produced by the young growing leaves, is the major agent-signal involved in the control of all aspects of xylem differentiation (Aloni, 1987a). The polar movement of auxin from the young leaves toward the roots through procambium, cambium, differentiating wood elements and parenchyma cells, triggers a complex sequence of changes that ultimately results in the formation of xylem tissue along the flow of auxin. The continuous flow of auxin results in a continuous pattern of xylem tissue that connects the leaves with the roots.

The normal polar flow of auxin from leaves to roots controls the polar longitudinal structure of the vascular and supporting elements along the plant axis. A horizontal diffusion of auxin around a wound induces the cells to transport auxin along a new horizontal polarity and results in the differentiation of xylem elements in the new horizontal polarity. Above a transverse wound, auxin can be forced to move in the pattern of close rings, which results in the differentiation of vessels in the form of close rings (Sachs, 1981). It has been well demonstrated in in vivo and in vitro systems that auxin is the stimulus and limiting factor that induces and controls tracheary element differentiation (Roberts et al., 1988; Aloni, 1990).

\section{Role of cytokinin in xylem differentia- tion}

Roots do not induce wood formation and need not be present to obtain xylem in stem tissues. However, the root apices are sources of inductive stimuli that promote xylem development. The major stimulus is cytokinin. Most of our knowledge concerning the role of cytokinin in xylem differentiation comes from studies in tissue culture (Roberts et al., 1988). Very little knowledge has been gained about the role of cytokinin in wood formation from studies done with intact plants.

The role of cytokinin was studied during secondary xylem fiber differentiation in intact young plants of Helianthus (Saks et al., 1984). Reducing the endogenous cytokinin supply, either by lateral root removal or by lowering the rate of transpiration, decreased the formation of secondary xylem fibers in the stem tissues of the plants. This decrease was considerably reversed by the addition of cytokinin to the roots. The application of cytokinin to intact plants via the roots promoted the differentiation of fibers in the secondary xylem in the hypocotyl. Cytokinin is required during the early stages of xylem fiber differentiation; later stages of fiber maturation can occur in the absence of cytokinin (Aloni, 1982).

\section{Role of gibberellin and fiber differentia- tion}

The differentiation of fibers is induced by stimuli originating in young and mature leaves. The inductive stimuli travel polarly along the plant axis and induce fibers along their pathway. The role of the leaves in the differentiation of fibers can be replaced by exogenous application of auxin combined with gibberellin. Both growth regulators also control the activity of the vascular cambium (Aloni, 1987b).

The combined presence of both hormones is a requirement for secondary xylem fiber development of Populus, where the applied gibberellin affects elongation of the xylem fibers but only in the presence of auxin (Digby and Wareing, 1966). In Phaseolus, a combination of an auxin (IAA) and a gibberellin $\left(G_{3}\right)$ induced xylem consisting almost entirely of secondary fibers (Hess and Sachs, 1972). 
Excision experiments, combined with wounding the stem of Coleus, have shown that $\mathrm{GA}_{3}$ can affect phloem fiber differentiation only in the presence of auxin. A combination of auxin and gibberellin applied in lanolin to decapitated and excised stems completely replaced the role of the leaves in phloem fiber differentiation, both qualitatively and quantitatively. When various combinations of both growth regulators were applied, high concentrations of IAA stimulated rapid differentiation of phloem fibers with thick secondary walls, whereas high levels of $\mathrm{GA}_{3}$ produced long fibers with thin walls (Aloni, 1979). The induction of long fibers by $\mathrm{GA}_{3}$ is compatible with findings of earlier studies on the effect of $\mathrm{GA}_{3}$ applied in the form of a spray to leaves of intact plants (Stant, 1963).

\section{Effect of ethylene on xylem differentia- tion}

Plant tissues synthesize ethylene in response to external pressures. Stress conditions, such as flooding, drought or bending of shoots, increase ethylene production in the stressed organs. These conditions affect wood differentiation in trees by regulating cambial activity and changing the quality and quantity of xylem production (Roberts and Miller, 1982; Roberts et al., 1988). For example, when Eucalyptus seedlings are placed in the horizontal position, they produce higher amounts of ethylene in their upper halves where the reaction wood is induced (Nelson and Hillis, 1978). Application of an ethylene-releasing compound (ethrel) to the stem of a tree induces more tracheid production in Pinus (Yamamoto and Kozlowski, 1987), and increases the number of vessels and reduces their diameter in Ulmus (Yamamoto et al., 1987). In both species, ethrel increased the amount of ray tissues in the treated region.

\section{Control of cell size and density}

Along a tree, there is a continuous increase in the size of individual wood elements from leaves to roots. This general increase is observed in both tracheid length and diameter, as one proceeds from branches to trunk and down into the roots of Sequoia trees (Bailey, 1958). Such an increase in tracheid size can also be observed in a transverse section in a stem or a branch of a tree. An increase proceeds outwards from the inner growth ring through a number of annual growth rings, until a constant size is usually attained. However, in the oldest stems of Pinus longaeva, tracheid length has steadily increased over the last 2200 years, with no signs of leveling off (Baas et al., 1986).

A similar polar pattern of gradual increase is found in vessel diameter and vessel length from twigs, down along the shoot and extending into the roots of Acer trees (Zimmermann and Potter, 1982). The basipetal increase in vessel diameter is associated with a decrease in vessel density; that is, the number of vessels per unit of transverse-sectional area. A similar pattern of basipetal increase in element length was reported for fibers in Fraxinus trees (Denne and Whitbread, 1978).

A hypothesis explaining the axial changes that occur in vascular element size and density from leaves to roots was proposed recently (Aloni and Zimmermann, 1983). Based on the polar flow of auxin as the major factor controlling vessel differentiation, the following 6-point hypothesis was developed: 1) basipetal polar flow of auxin from leaves to roots establishes a gradient of decreasing auxin concentration in this direction; 2) local 
structural or physiological obstruction of auxin flow results in a local increase in auxin concentration; 3) the distance from the source of auxin to the differentiating cells controls the amount of auxin flowing through the differentiating cells at a given time, thus determining the cells' position in the gradient; 4) the rate of conduit differentiation is positively correlated with the amount of auxin that the differentiating cells receive; consequently, the duration of the differentiation process increases from leaves to roots; 5) the final size of a conduit is determined by the rate of cell differentiation. Because cell expansion ceases after the secondary wall is deposited, rapid differentiation results in narrow vascular elements, while slow differentiation permits more cell expansion and therefore results in wide tracheary elements. Hence, decreasing auxin concentrations from leaves to roots leads to an increase in conduit size in this direction; 6) conduit density is controlled by, and is positively correlated with, the auxin concentration; consequentiy, vessel density decreases from leaves to roots.

Application of auxin to decapitated stems induces the differentiation of numerous tracheary elements immediately below the site of application and a progressively decreasing number of vascular elements with increasing distance below the auxin source. The auxin induces a substantial gradient of increasing vessel or tracheid diameter and decreases vessel density from the auxin source toward the root (Aloni and Zimmermann, 1983; Aloni, 1987b). Additional experimental evidence that supports the 6-point hypothesis was recently summarized (Roberts et al., 1988).

\section{Adaptation of the xylem to the tree's environment}

The safer conduit (vis-à-vis freezing and gas-induced cavitation or embolism) in the xylem of trees is the tracheid. Therefore, conifers, which are dependent upon tracheids for water transport, are very effective competitors in cold and dry habitats.

Hardwood trees, which are dependent upon vessels for water transport, show adaptation of their vessel system to their environment. In extreme habitats, dwarf trees develop and their xylem shows a high density of very narrow vessels. Such vascular systems, typical of extreme habitats, are deemed adaptive safety mechanisms against drought and freezing. The more numerous the vessels, the lower the probability is that water conduction would be seriously clamaged by an air embolism formed under water stress. Conversely, well-developed forest trees, which are typical in the tropics and rain forests, have vessels of very wide diameter, which affords maximal efficiency of water conduction (Zimmermann, 1983).

Aloni (1987b) suggested that the environment controls the size of the vessels through its control of the development, height and shape of the tree. The height and rate of branching determine the levels of the polar flow of auxin and probably other growth regulators along the tree. In order to explain how ecological conditions control the size and frequency of vessels and fibers in hardwood trees, the following vascular adaptation hypothesis (Aloni, 1987b) was proposed: 1) limiting conditions in the environment restrict the final size of the tree and result in small and suppressed shoots, whereas conditions which do not limit tree development allow the tree to reach its maximum potential height and size; 2) the duration of the growth period determines total tree development. In extreme and limiting habitats, the active growth period is relatively short and results in small trees, while stable and moderately comfortable conditions allow more growth activity throughout the year. Thus, more growth is 
accomplished and the result is large and well-developed trees; 3 ) the height of the tree and the rate of its branching determine gradients of auxin along the axis of the tree. An increase in tree height and decrease in branching increase the gradients of auxin from the young leaves (sources of auxin) to the lower parts of the stem. In small trees, which are typical of both dry and cold habitats, as well as locations where there is not enough soil for the roots, the distances from the young leaves to the roots are very short and no substantial gradient of auxin can be formed. Therefore, the levels of auxin are relatively high along these small trees and result in the differentiation of numerous very narrow vessels in the greatest densities, as expected from the 6-point hypothesis (Aloni and Zimmermann, 1983). These plants also have relatively small fibers with thick secondary walls (Aloni, 1979). On the other hand, in large trees, the very long distances from the young leaves to the roots enable a substantial decrease in auxin levels in the lower parts of the stem and in the roots, which results in the differentiation of very wide vessels in lower densities. These locations are also characterized by large fibers with relatively thin secondary walls.

Although some experimental evidence supports the above hypothesis (Aloni, 1987b; Robets et al., 1988) it should be tested in various experimental systems and under different growth conditions.

\section{Effect of gall-forming aphids on xylo- genesis}

A new phenomenon is described concerning the effect of insects' activity on wood formation in trees. It is shown that gallforming aphids influence xylem differentiation in the parts of a branch that are below a gall. Below a well-developed gall, more xylem differentiates and it is characterized by very wide vessels that develop in the earlywood as well as in the latewood (Aloni et al., 1989).

We have found experimentally that the wide vessels in Pistacia palaestinae, that differentiate below a gall formed by the aphid Bizongia pistaciae, substantially increase the ascent of sap towards the gall tissues, thus improving the water supply for the insects. We have also found that the differentiation of more wood with wide vessels below a gall is associated with an increase in auxin levels within the gall tissues (D.A. Katz and R. Aloni, unpublished observations).

\section{References}

Aloni R. (1979) Role of auxin and gibberellin in differentiation of primary phloem fibers. Plant Physiol. 63, 609-614

Aloni R. (1982) Role of cytokinin in differentiation of secondary xylem fibers. Plant. Physiol. 70, 1631-1633

Aloni R. (1987a) The induction of vascular tissues by auxin. In: Plant Hormones and Their Role in Plant Growth and Development. (Davies P.J., ed.), Martinus Nijhoff, Dordrecht, pp. 363374

Aloni R. (1987b) Differentiation of vascular tissues. Annu. Rev. Plant Physiol. 38, 179-204

Aloni R. (1990) Wood formation in deciduous hardwood trees. In: Physiology of Trees. (Raghavendra A.S., ed.), John Wiley \& Sons, New York, in press

Aloni R. \& Zimmermann M.H. (1983) The control of vessel size and density along the plant axis - a new hypothesis. Differentiation 24, 203-208

Aloni R., Katz D.A. \& Wool D. (1989) Effect of the gall-forming aphid Slavum wertheimae on the differentiation of xylem in branches of Pistacia atlantica. Ann. Bot. 63, 373-375

Baas P., Schmid R. \& Van Heuven B.J. (1986) Wood anatomy of Pinus longaeva (bristlecone pine) and the sustained length-on-age increase 
of its tracheids. Int. Assoc. Wood. Anat. Bull. (NS) 7, 221-228

Bailey I.W. (1958) The structure of tracheids in relation to the movement of liquids, suspensions and undissolved gases. In: The Physiology of Forest Trees. (Thimann K.V., ed.), Ronald, New York, pp. 71-82

Denne M.P. \& Whitbread V. (1978) Variation of fibre length within tree of Fraxinus excelsior. Can. J. For. Res. 8, 253-260

Digby J. \& Wareing P.F. (1966) The effect of applied growth hormones on cambial division and the differentiation of the cambial derivative. Ann. Bot. 30, 539-548

Hess T. \& Sachs T. (1972) The influence of a mature leaf on xylem differentiation. New Phytol. 71, 903-914

Nelson N.D. \& Hillis W.E. (1978) Ethylene and tension wood formation in Eucalyptus gomphocephala. Wood Sci. Technol. 12, 309-315

Roberts L.W. \& Miller A.R. (1982) Ethylene and xylem differentiation. What's New Plant Physiol. $13,13-16$

Roberts L.W., Gahan P.B. \& Aloni R. (1988) Vascular differentiation and plant growth regulators. In: Springer Series in Wood Science. (Timeli T.E., ed.), Springer Verlag, Berlin
Sachs T. (1981) The control of the patterned differentiation of vascular tissues. Adv. Bot. Res. 9, 152-255

Saks Y., Feigenbaum P. \& Aloni R. (1984) Regulatory effect of cytokinin on secondary xylem fiber formation in an in vivo system. Plant Physiol. 76, 638-642

Stant M.Y. (1963) The effect of gibberellic acid on cell width and cell wall of some phloem fibres. Ann. Bot. 27, 185-196

Yamamoto F. \& Kozlowski T.T. (1987) Effect of ethrel on growth and stem anatomy of Pinus halepensis seedlings. Int. Assoc. Wood. Anat. Bull. (NS) 8, 11-20

Yamamoto F., Angeles G. \& Kozlowski T.T. (1987) Effect of ethrel on stem anatomy of Ulmus americana seedlings. Int. Assoc. Wood. Anat. Bull. (NS) 8, 3-10

Zimmermann M.H. (1983) Xylem structure and the ascent of sap. In: Springer Series in Wood Science (Timell T.E., ed.), Springer Verlag, Berlin

Zimmermann M.H. \& Potter D. (1982) Vessellength distribution in branches, stem and roots of Acer rubrum L. Int. Assoc. Wood. Anat. Bull. (NS) 3, 103-109 